The Astronomical JournaL, 115:1418-1422, 1998 April

(C) 1998. The American Astronomical Society. All rights reserved. Printed in U.S.A.

\title{
A BLIND TEST OF PHOTOMETRIC REDSHIFT PREDICTION ${ }^{1}$
}

\author{
David W. Hogg ${ }^{2,3,4}$ Judith G. Cohen, ${ }^{5}$ Roger Blandford,${ }^{2}$ Stephen D. J. Gwyn, ${ }^{6}$ F. D. A. Hartwick, ${ }^{6}$ \\ B. Mobasher, ${ }^{7}$ Paula Mazzei ${ }^{8}$ Marcin Sawicki ${ }^{9}$ Huan Lin, ${ }^{9}$ H. K. C. Yee,${ }^{9}$ ANDrew J. Connolly,${ }^{10}$ \\ Robert J. BrunNer, ${ }^{10}$ IstVan Csabai,${ }^{10,11}$ MarK Dickinson,${ }^{10,12,13}$ Mark U. SubbaRao, ${ }^{10}$ \\ AleXANDer S. SZAlay, ${ }^{10}$ Alberto FernándeZ-Soto, ${ }^{14,15}$ \\ KENNETH M. LANZETTA, ${ }^{15}$ AND AMOS YAHIL ${ }^{15}$ \\ Received 1997 July 17; revised 1998 January 7
}

\begin{abstract}
Results of a blind test of photometric redshift predictions against spectroscopic galaxy redshifts obtained in the Hubble Deep Field with the Keck Telescope are presented. The best photometric redshift schemes predict spectroscopic redshifts with a redshift accuracy of $\Delta z<0.1$ for more than $68 \%$ of sources and with $\Delta z<0.3$ for $100 \%$, when single-feature spectroscopic redshifts are removed from consideration. This test shows that photometric redshift schemes work well, at least when the photometric data are of high quality and when the sources are at moderate redshifts.
\end{abstract}

Key words: galaxies: distances and redshifts - galaxies: photometry - methods: miscellaneous techniques: photometric - techniques: spectroscopic

\section{INTRODUCTION}

Photometric redshift prediction techniques, in which galaxy redshifts are estimated using only broadband photometric information, promise the acquisition of large numbers of redshifts of very faint sources with comparatively little telescope time, at least relative to performing a spectroscopic survey to comparable depth. The idea dates

\footnotetext{
${ }^{1}$ Based on observations with the NASA/ESA Hubble Space Telescope, obtained at the Space Telescope Science Institute, which is operated by the Association of Universities for Research in Astronomy (AURA), Inc., under NASA contract NAS 5-26555; at the W. M. Keck Observatory, which is operated jointly by the California Institute of Technology and the University of California; and at Kitt Peak National Observatory, which is operated by AURA, Inc., under cooperative agreement with the National Science Foundation.

${ }^{2}$ Theoretical Astrophysics, Mail Code 130-33, California Institute of Technology, Pasadena, CA 91125;rdb@tapir.caltech.edu.

${ }^{3}$ Current address: School of Natural Sciences, Institute for Advanced Study, Olden Lane, Princeton, NJ 08540; hogg@ias.edu.

${ }^{4}$ Hubble Fellow.

${ }^{5}$ Palomar Observatory, Mail Code 105-24, California Institute of Technology, Pasadena, CA 91125; jlc@astro.caltech.edu.

${ }^{6}$ Department of Physics and Astronomy, University of Victoria, Box 3055, Victoria, BC V8W 3P6, Canada; gwyn@uvastro.phys.uvic.ca, hartwick@uvastro.phys.uvic.ca.

${ }^{7}$ Astrophysics Group, Blackett Laboratory, Imperial College, Prince Consort Road, London SW7 2BZ, England, UK; b.mobasher@ic.ac.uk

${ }^{8}$ Osservatorio Astronomico, vicolo dell'Osservatorio 5, I-35122 Padova, Italy; mazzei@astrpd.pd.astro.it.

${ }^{9}$ Department of Astronomy, University of Toronto, Toronto, ON M5S 3H8, Canada; sawicki@astro.utoronto.ca, lin@astro.utoronto.ca, hyee@astro.utoronto.ca.

${ }^{10}$ Department of Physics and Astronomy, Johns Hopkins University, 3400 North Charles Street, Baltimore, MD 21218; ajc@pha.jhu.edu, rbrunner@pha.jhu.edu, csabai@pha.jhu.edu, subbarao@pha.jhu.edu, szalay@pha.jhu.edu.

${ }^{11}$ Department of Physics, Eötvös University, H-1088 Budapest, Hungary.

${ }^{12}$ Space Telescope Science Institute, 3700 San Martin Drive, Baltimore, MD 21218; med@stsci.edu.

${ }^{13}$ Alan C. Davis Fellow.

${ }^{14}$ Current address: Department of Astrophysics and Optics, School of Physics, University of New South Wales, Sydney, NSW 2052, Australia; fsoto@edwin.phys.unsw.edu.au.

15 Department of Physics and Astronomy, State University of New York at Stony Brook, Stony Brook, NY 11794-2100; lanzetta@sbast4.ess.sunysb.edu, ayahil@sbast4.ess.sunysb.edu.
}

back to Baum (1962), who used nine-band photoelectric photometry to (essentially) locate the $4000 \AA$ break in elliptical galaxies. Loh \& Spillar (1986) generalized this technique, with six-band CCD photometry, to apply to a wide range of galaxy types. With only four photographic bands, Koo (1985) was able to estimate redshifts from colors after plotting lines of constant redshift on color-color plots.

In the astronomical community, there is some perceived skepticism that photometric redshift estimation schemes are reliable or have been tested fairly. This skepticism may not appear in the literature or be well justified by demonstrated failings in photometric redshift techniques; however, we feel that it is strong enough to motivate a blind test of the methods. In this paper, the results of such a test are presented. The test was administered by three of us (D. W. H., J. G. C., R. B.) with no stake in the outcome.

For data, the imaging and spectroscopy of the Hubble Deep Field (HDF; Williams et al. 1996) was chosen. The reasons for this include that it has been imaged to enormous depth in the F300W, F450W, F606W, and F814W bandpasses (close to $U, B, V$ or $R$, and $I$ ) with the Hubble Space Telescope (HST); that it has been imaged from the ground in the near-infrared $J, H$, and $K$ bandpasses (Hogg et al. 1997b; Dickinson et al. 1998); and that there are various spectroscopic surveys underway in the field (Cohen et al. 1996; Steidel et al. 1996; Lowenthal et al. 1997) that can be used to train or develop photometric prediction methods.

There are three respects in which this is not the most stringent test on the photometric redshifts. The first is that the number of sources is small. Only 27 new sources in the HST image of the HDF had spectroscopic observations by the time the blind test was announced, so only this sample was available for the test. After reduction and analysis, only 21 of these have high-quality redshift determinations. The second is that the observational concentration on the HDF has been so intense that the photometric database is of much higher quality than that of any other existing field or any likely to exist in the near future. The third is that the spectroscopic redshifts used in the blind test are taken from a magnitude-limited survey of normal galaxies (Cohen et al. 1996, 1998) underway with the LRIS instrument (Oke et al. 1995) on the Keck Telescope. This survey, like most of its 
type, has difficulty identifying sources with redshifts in the range $1.5<z<2.5$ because of the lack of strong spectral features in the visual region free of strong night-sky emission. So the present work cannot test photometric redshift prediction techniques in this crucial redshift range, where perhaps they are most promising as a revolutionary tool in cosmology. On the other hand, this test is in one respect more stringent than previous tests: the sources employed for this blind test are on average fainter than those in the "training set," which is primarily the 1996 HDF redshifts (Cohen et al. 1996).

\section{DATA AND BLIND-TEST PROCEDURE}

During the 1997 February 6 and 7 runs on the Keck II Telescope, 27 sources in the $H S T$-imaged region of the HDF were observed spectroscopically with the LRIS instrument as part of the survey of Cohen et al. $(1996,1998)$. The positions but not redshifts of the 27 sources were announced by electronic mail to the HDF follow-up community on 1997 March 17, along with a call for photometric redshift predictions. The sources are listed in Table 1, along with chip numbers (in the HST HDF images), $(x, y)$-positions (in the "version 2 " reductions of the HST HDF data), magnitudes, and spectroscopic redshifts $z$ based on a fastpass reduction of the spectra. These and other HDF redshifts, along with details of selection and observing, will be

TABLE 1

SOURCES

\begin{tabular}{|c|c|c|c|c|}
\hline $\mathrm{Name}^{\mathrm{a}}$ & $\begin{array}{c}x, y^{\mathbf{b}} \\
\text { (pixels) }\end{array}$ & $\begin{array}{c}\mathrm{F} 606 \mathrm{~W}^{\mathrm{c}} \\
(\mathrm{mag})\end{array}$ & $\underset{(\mathrm{mag})}{\mathrm{F} 606 \mathrm{~W}-\mathrm{F} 814 \mathrm{~W}^{\mathrm{c}}}$ & $z^{\mathrm{d}}$ \\
\hline Н36431_1148.. & 1554,1582 & 24.1 & 1.7 & \\
\hline H36448_1200.. & 1385,1199 & 23.8 & 0.8 & 0.457 \\
\hline H36461_1246...... & 404,535 & 23.9 & 1.9 & \\
\hline H36465_1203.. & 1437,890 & 24.7 & 0.8 & 0.454 \\
\hline H36469_1422 ...... & 2030,792 & 20.8 & 1.3 & 0.000 \\
\hline H36471_1414...... & 1831,753 & 24.5 & 1.4 & 0.609 \\
\hline H36477_1232..... & 831,400 & 24.2 & 1.2 & 0.959 \\
\hline H36483_1426.. & 2032,1060 & 19.8 & 0.6 & 0.139 \\
\hline H36483_1214 ...... & 1307,514 & 24.3 & 1.3 & 0.962 \\
\hline H36483_1249...... & 478,148 & 22.8 & 2.4 & 0.000 \\
\hline H36487_1318. & 434,446 & 24.0 & 1.1 & 0.753 \\
\hline H36492_1148 ..... & 1961, 599 & 24.2 & 1.6 & 0.961 \\
\hline H36503_1418 ...... & 1709,1307 & 24.2 & 1.2 & 0.819 \\
\hline H36508_1250...... & 521,634 & 24.3 & 0.8 & $0.40:$ \\
\hline H36519_1332. & 527,1096 & 24.0 & 1.3 & 0.39 : \\
\hline H36520_1209.. & 294,1667 & 23.6 & 0.8 & 0.457 \\
\hline H36520_1400...... & 1184,1394 & 23.8 & 1.0 & 0.559 \\
\hline H36528_1405...... & 1220,1574 & 24.2 & 1.2 & 0.498 \\
\hline H36536_1417 ..... & 1451,1826 & 24.1 & 0.9 & 0.517 \\
\hline H36540_1245... & 980,981 & 24.1 & 1.9 & 0.000 \\
\hline H36541_1354.... & 886,1662 & 23.5 & 1.3 & 0.850 \\
\hline H36554_1311 ...... & 1463,489 & 24.1 & 2.1 & 1.315: \\
\hline H36555_1402..... & 979,1971 & 24.2 & 1.3 & 0.564 \\
\hline H36567_1252. & 1481,1009 & 25.1 & 1.7 & 1.235 \\
\hline H36569_1258.... & 1570,892 & 24.3 & 1.1 & 0.520 \\
\hline H36593_1255 ...... & 1928,1113 & 24.3 & 2.3 & 0.000 \\
\hline H37016_1225 ..... & 2009,1967 & 24.5 & 1.1 & 0.974: \\
\hline
\end{tabular}

a Sources are named by the convention that, for example, the source at $12^{\mathrm{h}} 37^{\mathrm{m}} 01^{\mathrm{s}} 6,+62^{\circ} 12^{\prime} 25^{\prime \prime}$ (J2000.0) is called "H37016_1225."

b Positions are given in pixels in the appropriate chip in the "version 2 " reduction of the HST HDF data.

' All magnitudes are measured through 1".5 diameter apertures.

d Redshifts $z$ are from Cohen et al. 1998. Sources without a successfully measured spectroscopic redshift are indicated with ellipses. Sources with $z=0.000$ are stars. Redshifts based on a single feature are marked with a colon. published in Cohen et al. (1998). Briefly, the sources all have $R<24 \mathrm{mag}$ and were spectroscopically observed with the same instrument setup as that described in Cohen et al. (1996). The magnitudes in Table 1 are Vega-relative and measured through 1".5 diameter apertures centered on the sources; they are provided only as rough flux estimates. Two sources have redshifts based on a single emission line assumed to be the [O II $] 3727 \AA$ line, and two have redshifts based on a weak break assumed to be the $4000 \AA$ break. These four spectroscopic redshifts are deemed less certain, and indicated in Table 1 and the results. Details of the spectroscopic analysis will be presented by Cohen et al. (1998).

By the close of the deadline on 1997 April 25, six photometric redshift groups had submitted predictions. After the results were presented at a meeting in 1997 May (Hogg, Cohen, \& Blandford 1997a), five groups agreed to participate in this summary paper.

\section{PREDICTION SCHEMES}

The photometric prediction schemes for the participating groups are described in this section, listed in the order in which the entries to the blind test were submitted (see Table 2).

\subsection{Victoria}

The Victoria method (Gwyn \& Hartwick 1996) uses the standard template-fitting method described in Gwyn (1997), although with modified templates. A spectral energy distribution (SED) is derived from the UBRI photometry of the HDF for each galaxy. This SED is compared with a series of redshifted templates. Two sets of templates are used: the model spectra of Bruzual \& Charlot (1993, hereafter BC) and the empirical spectra of Coleman, $\mathrm{Wu}, \&$ Weedman (1980, hereafter CWW; as extended by Ferguson \& McGaugh 1994). The former (BC) spectra have the advantage that they extend further into the ultraviolet and consequently can be used at higher redshifts. The latter (CWW) spectra were found to produce more accurate redshifts where they can be used (that is, at low redshift). Both sets of templates are corrected for intergalactic absorption using the prescription of Madau (1995). Use of the CWW templates and correction for intergalactic absorption represent two important improvements over the technique employed in Gwyn \& Hartwick (1996). As a first cut, a photometric redshift is determined by comparing the observed SED with the redshifted BC templates. Then, if this redshift is $z<1.5$, it is discarded in favor of a photometric redshift determined using the CWW templates.

\subsection{Imperial College}

The photometric redshifts of the Imperial College group are estimated by comparing observed SEDs with model SEDs for different populations of galaxies, shifted to different redshifts. This is performed in the following steps: (1) The observed SEDs of individual galaxies are established, using the $U$-, $B-, V$-, and $I$-band observations of the HDF. The $U$-band data are crucial for locating the $4000 \AA$ break. (2) Model SEDs corresponding to four template spectra (elliptical, spiral, irregular, and starburst) are constructed following recipes described by Mazzei, De Zotti, \& Xu (1994) and Mazzei, Curir, \& Bonoli (1995). These SEDs are extended from the far-ultraviolet to $1 \mu \mathrm{m}$ wavelengths and incorporate stellar emission, internal extinction, and re- 
TABLE 2

REDShIFT PREDICTIONS

\begin{tabular}{|c|c|c|c|c|c|c|c|}
\hline Name & Spectroscopic & Victoria & Imperial & $\begin{array}{l}\text { Toronto } \\
\text { Four-Color }\end{array}$ & $\begin{array}{c}\text { Toronto } \\
\text { Seven-Color }\end{array}$ & Johns Hopkins & Stony Brook \\
\hline H36431_1148...... & $\ldots$ & 0.900 & 0.600 & 1.05 & 1.10 & 1.22 & 1.04 \\
\hline H36448_1200...... & 0.457 & 0.480 & 0.490 & 0.40 & 0.50 & 0.56 & 0.36 \\
\hline H36461_1246...... & $\ldots$ & 0.765 & 0.675 & 0.90 & 0.65 & 0.75 & 0.60 \\
\hline H36465_1203...... & 0.454 & 0.405 & 0.175 & 0.50 & 0.50 & 0.35 & 0.48 \\
\hline H36469_1422..... & 0.000 & 0.000 & $\ldots$ & $\ldots$ & $\ldots$ & $\ldots$ & 0.000 \\
\hline H36471_1414...... & 0.609 & 0.660 & 0.650 & 0.65 & 0.65 & $\ldots$ & 0.68 \\
\hline H36477_1232..... & 0.959 & 0.810 & 0.750 & 0.90 & 1.00 & 1.01 & 1.04 \\
\hline H36483_1214..... & 0.962 & 0.945 & 0.800 & 1.15 & 1.10 & 0.93 & 1.12 \\
\hline H36483_1249...... & 0.000 & $\ldots$ & $\ldots$ & $\ldots$ & $\ldots$ & $\ldots$ & 0.000 \\
\hline H36483_1426...... & 0.139 & $\ldots$ & $\ldots$ & $\ldots$ & $\ldots$ & $\ldots$ & 0.12 \\
\hline H36487_1318...... & 0.753 & 0.780 & 0.750 & 0.70 & 0.70 & 0.75 & 0.80 \\
\hline H36492_1148...... & 0.961 & 0.840 & 0.800 & 0.95 & 0.95 & 0.90 & 1.00 \\
\hline H36503_1418...... & 0.819 & 0.780 & 0.775 & 0.95 & 0.80 & 0.86 & 0.84 \\
\hline H36508_1250..... & $0.40:$ & 0.525 & 0.650 & 1.95 & 0.40 & 0.41 & 0.56 \\
\hline H36519_1332...... & 0.39: & 0.840 & 0.750 & 1.10 & 1.00 & 0.98 & 0.96 \\
\hline H36520_1209..... & 0.457 & 0.435 & 0.650 & 0.50 & 0.45 & 0.37 & 0.52 \\
\hline H36520_1400...... & 0.559 & 0.540 & 0.650 & 0.55 & 0.55 & 0.53 & 0.52 \\
\hline H36528_1405...... & 0.498 & 0.690 & 0.650 & 0.55 & 0.60 & 0.62 & 0.64 \\
\hline H36536_1417...... & 0.517 & 0.450 & 0.600 & 0.45 & 0.35 & 0.46 & 0.44 \\
\hline H36540_1245...... & 0.000 & 0.000 & $\ldots$ & 0.000 & 0.000 & 0.000 & 0.60 \\
\hline H36541_1354...... & 0.850 & 0.795 & 0.725 & 1.00 & 0.95 & 0.86 & 1.00 \\
\hline H36554_1311...... & 1.315: & 1.095 & 0.750 & 1.05 & 0.95 & 1.17 & 0.88 \\
\hline H36555_1402...... & 0.564 & 0.570 & 0.650 & 0.60 & 0.30 & 0.56 & 0.68 \\
\hline H36567_1252...... & 1.235 & 1.020 & 1.400 & 1.95 & 1.25 & 0.96 & 0.92 \\
\hline H36569_1258..... & 0.520 & 0.510 & 0.200 & 0.50 & 0.55 & 0.35 & 0.56 \\
\hline H36593_1255...... & 0.000 & 0.000 & $\ldots$ & $\ldots$ & $\ldots$ & 0.000 & 0.56 \\
\hline H37016_1225...... & 0.974: & 1.600 & 1.450 & $\ldots$ & $\cdots$ & $\ldots$ & 1.00 \\
\hline
\end{tabular}

emission by dust. At the rest frame these fit the observed SEDs of local galaxies of their respective type. These models, computed using the evolutionary population synthesis technique, assume a star formation rate and the initial mass function, permitting calculation of the template SEDs at different redshifts. The model parameters are then constrained by fitting the SEDs of a sample of 53 galaxies in the HDF with spectroscopic redshifts (Cohen et al. 1996; Steidel et al. 1996). The rms scatter between the photometric redshifts and their spectroscopic counterparts (Cohen et al. 1996; Steidel et al. 1996) is found to be 0.11 . The final models correspond to formation redshifts $z_{f}=5(13 \mathrm{Gyr})$ for ellipticals, $2(10-11 \mathrm{Gyr})$ for spirals, $1(0.8-0.9 \mathrm{Gyr})$ for irregulars, and $5(13 \mathrm{Gyr})$ for starbursts (taking $H_{0}=50 \mathrm{~km}$ $\mathrm{s}^{-1} \mathrm{Mpc}^{-1}$ and $q_{0}=0.5$ ). A detailed description of the model SEDs is given in Mobasher, Mazzei, \& RowanRobinson (1998). (3) A matrix with elements consisting of the template SEDs for the four types of galaxies, shifted in redshift space, is generated with the evolutionary models. The redshift and spectral type corresponding to the template SED closest to the observed SED are associated with each galaxy. A detailed description of the technique is given in Mobasher et al. (1996).

In the original blind test, because of a coordinate system discrepancy, the list of sources submitted by the Imperial College group did not match the list presented in the call for entries to the test. After the results were presented, the Imperial College group submitted photometric redshifts for the correct list of sources. In principle, this compromises the blindness of the test, although no modification was made to the Imperial College photometric redshift prediction technique between the first and second submissions.

\subsection{Toronto Four-Color}

The Toronto four-color predictions are the redshift estimates of Sawicki, Lin, \& Yee (1997). This method uses the

TABLE 3

RESULTS

\begin{tabular}{|c|c|c|c|c|}
\hline \multirow[b]{2}{*}{ INSTITUTION (SUBMITTER) } & \multicolumn{2}{|c|}{$|\Delta z| \leq 0.1$} & \multicolumn{2}{|c|}{$|\Delta z| \leq 0.3$} \\
\hline & Total & Good $^{a}$ & Total & Good $^{\mathrm{a}}$ \\
\hline University of Victoria (S. D. J. G.) & $15 / 23$ & $15 / 19$ & $21 / 23$ & $19 / 19$ \\
\hline Imperial College (B. M.) ..... & $7 / 20$ & $7 / 16$ & $16 / 20$ & $15 / 16$ \\
\hline University of Toronto Four-Color (M. S.) & $13 / 20$ & $13 / 17$ & $17 / 20$ & $16 / 17$ \\
\hline University of Toronto Seven-Color (M. S.) ${ }^{\mathbf{b}}$. & $14 / 20$ & $13 / 17$ & $18 / 20$ & $17 / 17$ \\
\hline Johns Hopkins University (A. J. C. $)^{\mathrm{b}}$.. & $13 / 20$ & $12 / 17$ & $19 / 20$ & $17 / 17$ \\
\hline SUNY Stony Brook (A. F.-S.) ${ }^{\mathrm{b}, \mathrm{c}} \ldots \ldots$ & $15 / 25$ & $14 / 21$ & $20 / 25$ & $18 / 21$ \\
\hline
\end{tabular}

a The "Good" columns do not include single-feature spectroscopic redshifts (see text).

${ }^{b}$ Makes use of additional ground-based infrared data.

c The Stony Brook results include two sources that are stars, but are predicted to have redshifts $z \sim 0.6$. Removing these sources, the Stony Brook numbers become 15/23, 14/19, $20 / 23$, and $18 / 19$, respectively. 


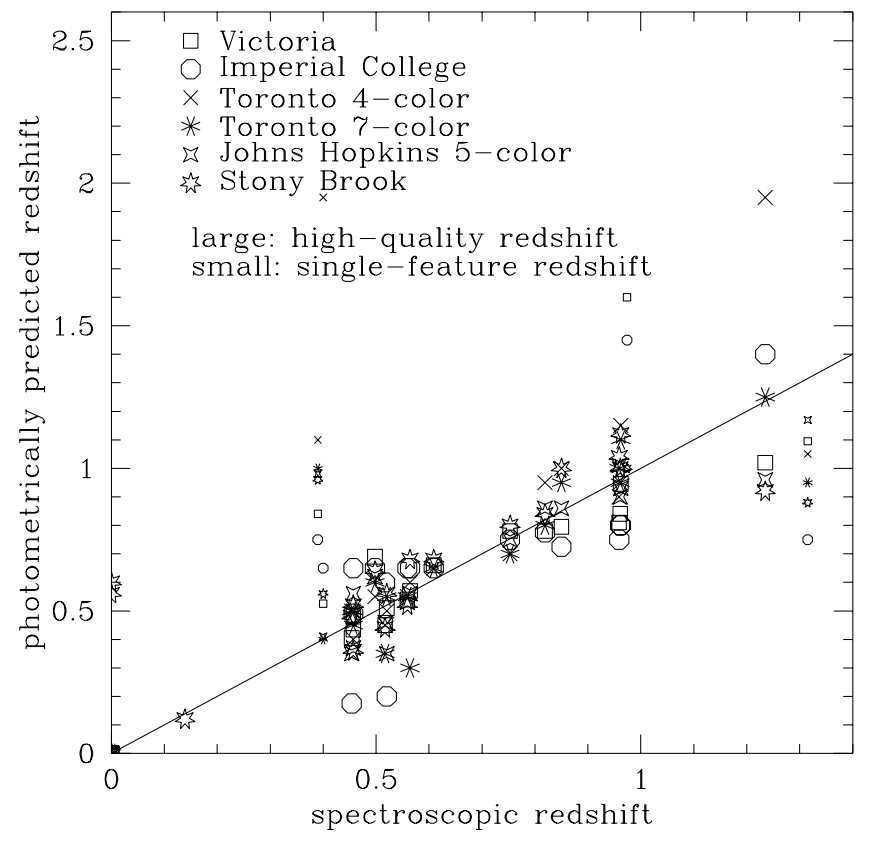

FIG. 1.-Photometric vs. spectroscopic redshifts for the sample. Smaller symbols are used for single-feature spectroscopic redshifts (see text).

four HST filters to measure the colors of each galaxy, and automatic morphological classification to weed out stars. Redshifts are determined by comparing the observed colors with those of a set of model templates. The templates were computed on the basis of the empirical $z \sim 0$ spectral energy distributions of CWW; these SEDs have been augmented and extended into the ultraviolet by grafting on the spectral shapes of $\mathrm{BC}$; they were then UV-suppressed using Madau's (1995) prescription for high-redshift intergalactic $\mathrm{H}$ continuum and line blanketing; the resultant SEDs were convolved with instrumental response curves to produce model colors. For each object, the best-matching template and, hence, redshift and spectral type are chosen by $\chi^{2}$ minimization of the observed fluxes with respect to the model colors.

\subsection{Toronto Seven-Color}

The Toronto seven-color method is identical to the Toronto four-color method, except that (1) three infrared filters ( $I, J$, and $K$; Dickinson et al. 1998) are added, and (2) for the purposes of measuring galaxy colors, the seeing in the four HST images is degraded to make them compatible with the ground-based infrared data. The inclusion of the infrared data decreases the confusion between the various spectral breaks - in particular, the Balmer and the $912 \AA$ break - and thereby increases the accuracy of photometric redshifts.

\subsection{Johns Hopkins}

Because the uncertainties in using low-redshift spectral energy distributions are large, particularly in the ultraviolet, the Johns Hopkins method adopts a more empirical approach to the problem of photometric redshift prediction. Using recent spectroscopic redshift survey data as a training set, an empirical relation between the photometric properties of galaxies (their fluxes and colors) and their redshifts is derived. For the HDF a correlation between the optical and follow-up near-infrared $J$-band photometry (Dickinson et

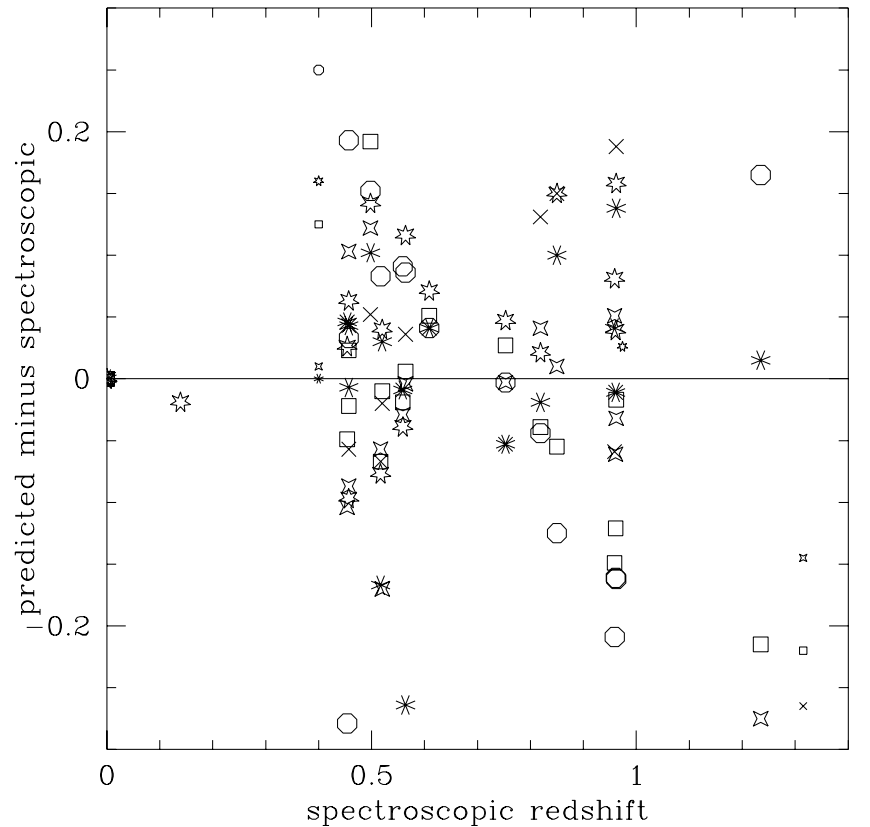

FIG. 2.- Redshift differences, predicted minus spectroscopic, expanded to show only those predictions with differences $\Delta z<0.3$. Symbols are as in Fig. 1.

al. 1998) and redshift is derived. A third-order polynomial in the F300W, F450W, F606W, F814W, and $J$ passbands is fitted to the 73 galaxies with previously published spectroscopic data (Cohen et al. 1996; Steidel et al. 1996; Lowenthal et al. 1997). This relation is applied to those galaxies without spectroscopic redshifts. The inclusion of the near-infrared data significantly improves the precision of the photometric redshift relation, primarily for $z>1$, because the $\sim 4000 \AA$ break moves out of the optical spectral region and into the near-infrared at these redshifts. For star-forming galaxies, the ultraviolet continuum from Ly $\alpha$ $(1215 \AA)$ to $\sim 3000 \AA$ is relatively devoid of strong features, and consequently there is little information in the optical photometry from which to estimate redshifts between $1<z<2$. Incorporating the $J$ band alone extends the redshift interval over which reliable photometric redshifts can be obtained to $z \sim 2$. Full details, discussing the derivation and application of this photometric redshift technique, can be found in Connolly et al. (1995), SubbaRao et al. (1996), Brunner et al. (1997), and Connolly et al. (1997).

\subsection{Stony Brook}

The Stony Brook technique is essentially that of Lanzetta, Yahil, \& Fernández-Soto (1996), but takes advantage of ground-based near-infrared images of the HDF (Dickinson et al. 1998). A more detailed description of this work is presented elsewhere (Fernández-Soto, Lanzetta, \& Yahil 1998). Flux measurements of every object in the HDF optical images are performed using identical apertures on all four filters. For every object, its aperture is given by a mask determined by the set of pixels that the SExtractor program (Bertin \& Arnouts 1996) assigns to it in the F814W image. By convolving the F814W image of each source within its mask aperture with the appropriate near-infrared ( $J, H$, or $K$ band) PSF and normalizing, a model of the infrared images can be created. This procedure makes the assumption that morphology is not a strong function of wavelength. The model has as parameters the fluxes of every 


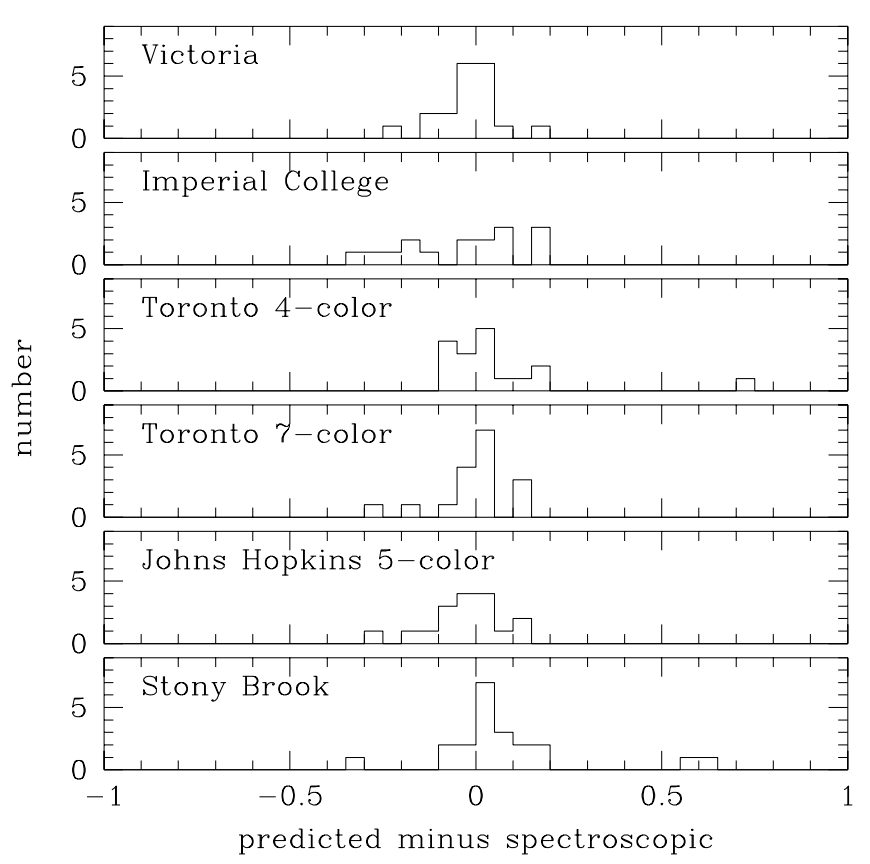

FIG. 3.-Histogram of redshift differences for each group, excluding those sources with single-feature spectroscopic redshifts.

object in the $J, H$, and $K$ bands. This model is fitted to the data to provide matched near-infrared fluxes for every object together with their errors. These fluxes are compared with the expected fluxes from the model SEDs (Lanzetta et al. 1996; the SEDs are essentially those of Coleman et al. 1980), extended to the near-infrared according to the models of BC. Intergalactic $\mathrm{H}$ I absorption was taken into account as described in Lanzetta et al. (1996). No galaxystar separation was performed, but obviously stellar sources were removed after visual inspection; this can lead to some misassignations of nonzero redshifts to stars.

\section{RESULTS}

Different groups make predictions for different total numbers of sources, because their catalogs are selected in different bands with different algorithms over different areas.

As Table 3 shows, all photometric redshift techniques surveyed here do well, with many sources in agreement with spectroscopy at the $\Delta z<0.1$ level. When only multifeature spectroscopic redshifts are considered, most techniques show better than $68 \%$ agreement at the $\Delta z<0.1$ level and all show $90 \%-100 \%$ agreement with spectroscopy at the $\Delta z<0.3$ level (when stars are removed from the Stony Brook catalog). Unfortunately, the spectroscopic sample does not include sources past $z=1.4$, so only lower redshifts are tested. The results of this study should be considered encouraging for those interested in using photometric redshifts to define spectroscopic samples and estimate luminosity functions at $0.4<z<1$, with the caveats that the photometric data here are of higher than average quality and that it will be necessary to perform studies with a larger number of redshifts to make a rigorous test and to characterize systematics. The results are shown graphically in a redshift-redshift diagram in Figure 1, in a redshift error-redshift diagram in Figure 2, and in redshift error histograms in Figure 3. No strong systematic discrepancies between photometric and spectroscopic redshifts are evident, although given the small number of sources only systematic effects larger than about 0.2 in redshift over the tested redshift range could be detected. For all groups the $95 \%$ error region in $\Delta z$ is more than twice the size of the $68 \%$; this provides some evidence that the discrepancies between photometric and spectroscopic redshifts are not Gaussian distributed.

The HDF team, led by Bob Williams, are thanked for planning, taking, reducing, and making public the beautiful HST images of the HDF, as are Len Cowie and the Hawaii group for confirming some originally uncertain redshifts. D. W. H. thanks the Canadian Institute for Theoretical Astrophysics for hospitality. Support from the NSF and NASA is gratefully acknowledged, including NSF grant AST 9529170, and HST data analysis grant AR-06337.12-94A and Hubble Fellowship grant HF-01093.01-97A, both from the Space Telescope Science Institute (operated by AURA, Inc., under NASA contract NAS 5-26555).

\section{REFERENCES}

Baum, W. A. 1962, in IAU Symp. 15, Problems of Extra-galactic Research, ed. G. C. McVittie (New York: Macmillan), 390

Bertin, E., \& Arnouts, S. 1996, A\&A, 117, 393

Brunner, R. J., Connolly, A. J., Szalay, A. S., \& Bershady, M. A. 1997, ApJ, 482, L21

Bruzual A., G., \& Charlot, S. 1993, ApJ, 405, 538 (BC)

Cohen, J. G., Cowie, L. L., Hogg, D. W., Songaila, A., Blandford, R., Hu, E. M., \& Shopbell, P. 1996, ApJ, 471, L5

Cohen, J. G., et al. 1998, in preparation

Coleman, G. D., Wu, C.-C., \& Weedman, D. W. 1980, ApJS, 43, 393 (CWW)

Connolly, A. J., Csabai, I., Szalay, A. S., Koo, D. C., Kron, R. C., \& Munn, J. A. 1995, AJ, 110, 2655

Connolly, A. J., Szalay, A. S., Dickinson, M., SubbaRao, M. U., \& Brunner, R. J. 1997, ApJ, 486, 11

Dickinson, M., et al. 1998, in preparation

Ferguson, H. C., \& McGaugh, S. S. 1994, ApJ, 440, 470

Fernández-Soto, A., Lanzetta, K. M., \& Yahil, A. 1998, in preparation

Gwyn, S. D. J. 1997, PASP, submitted

Gwyn, S. D. J., \& Hartwick, F. D. A. 1996, ApJ, 468, L77

Hogg, D. W., Cohen, J. G., \& Blandford, R. 1997a, in The Hubble Deep Field: Poster Papers, ed. M. Livio, S. M. Fall, \& P. Madau (Baltimore: STScI)

Hogg, D. W., Neugebauer, G., Armus, L., Matthews, K., Pahre, M. A., Soifer, B. T., \& Weinberger, A. J. 1997b, AJ, 113, 474

Koo, D. C. 1985, AJ, 90, 418

Lanzetta, K. M., Yahil, A., \& Fernández-Soto, A. 1996, Nature, 381, 759

Loh, E. D., \& Spillar, E. J. 1986, ApJ, 303, 154

Lowenthal, J. D., et al. 1997, ApJ, 481, 673

Madau, P. 1995, ApJ, 441, 18

Mazzei, P., Curir, A., \& Bonoli, C. 1995, AJ, 110, 559

Mazzei, P., De Zotti, G., \& Xu, C. 1994, ApJ, 422, 81

Mobasher, B., Mazzei, P., \& Rowan-Robinson, M. 1998, in preparation

Mobasher, B., Rowan-Robinson, M., Georgakakis, A., \& Eaton, N. 1996, MNRAS, 282, L7

Oke, J. B., et al. 1995, PASP, 107, 375

Sawicki, M. J., Lin, H., \& Yee, H. K. C. 1997, AJ, 113, 1

Steidel, C. C., Giavalisco, M., Dickinson, M., \& Adelberger, K. L. 1996, ApJ, 462, 17

SubbaRao, M. U., Connolly, A. J., Szalay, A. S., \& Koo, D. C. 1996, AJ, 112,929

Williams, R. E., et al. 1996, AJ, 112, 1335 